\title{
Wolfe Type Second Order Nondifferentiable Symmetric Duality in Multiobjective Programming over Cone with Generalized $(K, F)$-Convexity
}

\author{
A. K. Tripathy \\ Department of Mathematics, Trident Academy of Technology, Chandaka Industrial Estate, F2/A, Bhubaneswar, Odisha 751024, India \\ Correspondence should be addressed to A. K. Tripathy; arun_tripathy06@rediffmail.com
}

Received 2 January 2014; Accepted 6 February 2014; Published 8 April 2014

Academic Editors: F. Ding and F. Zirilli

Copyright @ 2014 A. K. Tripathy. This is an open access article distributed under the Creative Commons Attribution License, which permits unrestricted use, distribution, and reproduction in any medium, provided the original work is properly cited.

A new class of second order $(K, F)$ pseudoconvex function is introduced with example. A pair of Wolfe type second order nondifferentiable symmetric dual programs over arbitrary cones with square root term is formulated. The duality results are established under second order $(K, F)$ pseudoconvexity assumption. Also a Wolfe type second order minimax mixed integer programming problem is formulated and the symmetric duality results are established under second order $(K, F)$ pseudoconvexity assumption.

\section{Introduction}

A mathematical programming with two or more objective functions is called multiobjective programming. Often the several objectives are conflicting in nature. Pareto [1] studied multiobjective problems by reducing them to a single objective one. However, the problems were first explicitly defined and studied by Kuhn and Tucker [2]. They also proposed the definition of proper efficiency which was later modified by Geoffrion [3].

In mathematical programming, a pair of primal and dual programs is called symmetric if the dual of the dual is the primal problem. The duality in linear programming is symmetric. It is not so in nonlinear programming in general. Dorn [4], Dantzig et al. [5], and Mond [6] studied symmetric duality in nonlinear programming assuming the kernel function $f(x, y)$ to be convex in $x$ and concave in $y$. Subsequently, Mond and Weir [7] presented a distinct pair of symmetric dual nonlinear programs which admits the relaxation of the convexity/concavity assumption to pseudoconvexity/pseudoconcavity. Mond [6] initiated second order symmetric duality of Wolfe type in nonlinear programming and proved the duality theorems under second order convexity. Mangasarian [8] discussed second order duality in nonlinear programming under inclusion condition. Mond [6, page 93] and Mangasarian [8, page 609] also indicated possible computational advantages of the second order dual over the first order dual. This motivated several authors [3, $6,9-13$ ] in this field. Yang et al. [13] studied second order multiobjective symmetric dual programs and established the duality relations under $F$-convexity assumptions. Also Yang et al. [12] formulated a pair of Wolfe type second order nondifferentiable symmetric dual programs containing support function and presented the duality results under $F$ convexity.

Recently, Gulati et al. [14] studied Wolfe and MondWeir type second order symmetric duality over arbitrary cones and proved the duality results under generalized convexity assumption. Gulati and Geeta [15] studied MondWeir type second order symmetric duality in multiobjective programming over cones and established duality results under pseudoconvexity $/ K-F$ convexity assumption. Gulati and Verma [16] formulated a pair of Wolfe type nondifferentiable multiobjective symmetric duality and established the duality results under invexity assumption. Gupta and Kailey [17] formulated a pair of Wolfe type second order nondifferentiable multiobjective symmetric dual programs 
in which the objective function contains support function and proved the duality results under second order F-convexity assumption. Gupta and Kailey [18] presented second order multiobjective symmetric duality involving cone-convex functions. Saini and Gulati [19] presented a pair of Wolfe type nondifferentiable second order symmetric dual programs over arbitrary cones under second order $(K, F)$ convexity assumption.

In this paper, motivated by Saini and Gulati [19], a new class of second order $(K, F)$ pseudoconvex/second order $(K, F)$ strongly pseudoconvex function is introduced with example. A pair of Wolfe type second order nondifferentiable symmetric dual programs over arbitrary cone containing square root term is formulated. The duality results are established under second order $(K, F)$ pseudoconvexity assumption.

\section{Notation and Preliminaries}

The following convention for vectors in $R^{n}$ will be used:

$$
\begin{aligned}
& x<y \Longleftrightarrow x_{i}<y_{i}, \quad i=1,2, \ldots, n, \\
& x \leqq y \Longleftrightarrow x_{i} \leqq y_{i}, \quad i=1,2, \ldots, n, \\
& x \leq y \Longleftrightarrow x_{i} \leq y_{i}, \quad i=1,2, \ldots, n, \text { but } x \neq y .
\end{aligned}
$$

Definition 1. A set $C$ of $R^{n}$ is called a cone if, for each $x \in C$ and $\lambda \in R, \lambda \geq 0$, we have $\lambda x \in C$. Moreover, if $C$ is convex, then it is convex cone.

Definition 2. The positive polar cone $C^{*}$ of $C$ is defined as

$$
C^{*}=\left\{z \in R^{n} \mid x^{T} z \geq 0, \forall x \in C\right\} .
$$

Let $C_{1} \subset R^{n}, C_{2} \subset R^{m}$, and $K \subset R^{k}$ be closed convex cones with nonempty interiors having polars $C_{1}^{*}, C_{2}^{*}$, and $K^{*}$, respectively. Let $X \subseteq R^{n}$ and $Y \subseteq R^{m}$ be open and $X \times Y \subseteq R^{n} \times R^{m}$. Let $C_{1} \times C_{2} \subseteq X \times Y$.

A general multiobjective nonlinear programming problem can be expressed in the following form.

Primal (P).

$$
\begin{array}{ll}
\text { Minimize } & f(x)=\left\{f_{1}(x), f_{2}(x), \ldots, f_{k}(x)\right\} \\
\text { Subject to } & -g(x) \in Q, \quad x \in X,
\end{array}
$$

where $X \subseteq R^{n}$ is open,

$f: R^{n} \rightarrow R^{k}, g: R^{n} \rightarrow R^{l}, Q$ is a closed convex cone with nonempty interior in $R^{m}$.

Definition 3. A feasible point $\bar{x}$ is weakly efficient solution of (P) if there exist no other $x \in X$ such that $f(x)-f(\bar{x})<0$.

Definition 4. A feasible point $\bar{x}$ is efficient solution of (P) if there exist no other $x \in X$ such that $f(x)-f(\bar{x}) \leq 0$.

Definition 5. A function $F: X \times X \times R^{n} \rightarrow R$ is sublinear in its third argument if, for all $(x, u) \in X \times X$, (1) $F\left(x, u ; a_{1}+a_{2}\right) \leq F\left(x, u ; a_{1}\right)+F\left(x, u ; a_{2}\right)$, for all
$a_{1}, a_{2} \in R^{n}$

(2) $F(x, u ; \alpha a)=\alpha F(x, u ; a)$, for all $\alpha \in R_{+}$.

Definition 6. Let $f: R^{n} \rightarrow R^{k}$ be thrice differentiable function. $f$ is said to be second order $F$-pseudoconvex at $u \in X$, if $(x, p) \in X \times R^{n}$,

$$
\begin{aligned}
& F_{x, u}\left(\nabla_{u} f(u)+\nabla_{u u} f(u) p\right) \geq 0 \\
& \Longrightarrow f(x)-f(u)+\frac{1}{2} p^{T} \nabla_{u} f(u) p \geq 0 .
\end{aligned}
$$

Now, we are in position to give definition of second order $(K$, $F)$-pseudoconvex function and second order strongly $(K, F)$ pseudoconvex function.

Definition 7. The thrice differentiable function $f=\left(f_{1}, f_{2}\right.$, $\left.\ldots, f_{k}\right): R^{n} \rightarrow R^{k}$ is said to be second order $(K, F)$ pseudoconvex at $u \in X$, if $(x, p) \in X \times R^{n}$,

$$
\begin{gathered}
-\left(F_{x, u}\left(\nabla_{u} f_{1}(u)+\nabla_{u u} f_{1}(u) p_{1}\right), \ldots,\right. \\
\left.F_{x, u}\left(\nabla_{u} f_{k}(u)+\nabla_{u u} f_{k}(u) p_{1}\right)\right) \notin \operatorname{int} K \\
\Longrightarrow-\left(f_{1}(x)-f_{1}(u)+\frac{1}{2} p_{1}^{T} \nabla_{u} f_{1}(u) p_{1}, \ldots, f_{k}(x)\right. \\
\left.-f_{k}(u)+\frac{1}{2} p_{k}^{T} \nabla_{u} f_{k}(u) p_{k}\right) \notin \operatorname{int} K .
\end{gathered}
$$

Definition 8. The thrice differentiable function $f: R^{n} \rightarrow R^{k}$ is said to be second order strongly $(K, F)$ pseudoconvex at $u \in X$, if $(x, p) \in X \times R^{n}$,

$$
\begin{gathered}
-\left(F_{x, u}\left(\nabla_{u} f_{1}(u)+\nabla_{u u} f_{1}(u) p_{1}\right), \ldots,\right. \\
\left.F_{x, u}\left(\nabla_{u} f_{k}(u)+\nabla_{u u} f_{k}(u) p_{1}\right)\right) \notin \operatorname{int} K, \\
\Longrightarrow\left(f_{1}(x)-f_{1}(u)+\frac{1}{2} p_{1}^{T} \nabla_{u} f_{1}(u) p_{1}, \ldots, f_{k}(x)\right. \\
\left.-f_{k}(u)+\frac{1}{2} p_{k}^{T} \nabla_{u} f_{k}(u) p_{k}\right) \in \operatorname{int} K .
\end{gathered}
$$

Definition 9. $f$ is second order $(K, F)$ pseudoconcave, if $-f$ is second order $(K, F)$ pseudoconvex, and $f$ is second order strongly $(K, F)$ pseudoconcave, if $-f$ is second order strongly $(K, F)$ pseudoconvex function. 
Example 10. Let

$$
\begin{gathered}
K=\left\{(x, y) \mid-4 x \leq y \leq-\frac{x}{2}, x>0\right\} \\
f(x)=\left(f_{1}(x), f_{2}(x)\right)=\left(-x^{2}+x, e^{-x}\right), \quad p=1 \\
F_{x, u}(a)=a\left(x^{3}+u\right) \\
\left(\nabla_{u} f_{1}(u), \nabla_{u} f_{2}(u)\right) \\
=\left(-2 u+1,-e^{-u}\right), \\
\left(\nabla_{u u} f_{1}(u), \nabla_{u u} f_{2}(u)\right)=\left(-2, e^{-u}\right) \\
\left.\left(\nabla_{1}, a_{2}\right)=(u)+\nabla_{u u} f_{1}(u) p, \nabla_{u} f_{2}(u)+\nabla_{u u} f_{2}(u) p\right) \\
=(-2 u-1,0) ; \\
\left(F_{x, u}\left(a_{1}\right), F_{x, u}\left(a_{2}\right)\right)=(-2 u-1,0)\left(x^{3}+u\right) \\
\left(\begin{array}{c}
f_{1}(x)-f_{1}(u)+\frac{1}{2} p^{T} \nabla_{u u} f_{1}(u) p \\
f_{2}(x)-f_{2}(u)+\frac{1}{2} p^{T} \nabla_{u u} f_{2}(u) p
\end{array}\right) \\
=\left(-x^{2}+x+u^{2}-u-1, e^{-x}-\frac{1}{2} e^{-u}\right)
\end{gathered}
$$

Now at

$$
\left(F_{x, u}\left(a_{1}\right), F_{x, u}\left(a_{2}\right)\right) \geq 0 \Longrightarrow x \leq 0
$$

So at

$$
\left(\begin{array}{c}
f_{1}(x)-f_{1}(u)+\frac{1}{2} p^{T} \nabla_{u u} f_{1}(u) p, \\
f_{2}(x)-f_{2}(u)+\frac{1}{2} p^{T} \nabla_{u u} f_{2}(u) p
\end{array}\right) \geq 0 .
$$

So $f$ is not second order $F$ convex function.

Now at

$$
\begin{gathered}
u=0, x=1, \quad-\left(F_{x, u}\left(a_{1}\right), F_{x, u}\left(a_{2}\right)\right)=(1,0) \notin \operatorname{int} K \\
\left(f_{1}(x)-f_{1}(u)+\frac{1}{2} p^{T} \nabla_{u u} f_{1}(u) p, f_{2}(x)\right. \\
\left.-f_{2}(u)+\frac{1}{2} p^{T} \nabla_{u u} f_{2}(u) p\right) \\
=\left(-1, e^{-1}-\frac{1}{2}\right) \notin \operatorname{int} K .
\end{gathered}
$$

So $f$ is not second order strongly $(K, F)$ pseudoconvex. But

$$
\begin{aligned}
& -\left(f_{1}(x)-f_{1}(u)+\frac{1}{2} p^{T} \nabla_{u u} f_{1}(u) p, f_{2}(x)\right. \\
& \left.-f_{2}(u)+\frac{1}{2} p^{T} \nabla_{u u} f_{2}(u) p\right) \\
& =\left(1,-e^{-1}+\frac{1}{2}\right) \notin \operatorname{int} K .
\end{aligned}
$$

So $f$ is second order $(K, F)$ pseudoconvex.
Now we can define second order $(K, F)$ pseudoconvexity for a multiobjective function:

$$
f=\left(f_{1}, f_{2}, \ldots, f_{k}\right): R^{n} \times R^{m} \longrightarrow R^{k}
$$

Definition 11. A thrice differentiable function $f=\left(f_{1}, f_{2}\right.$, $\left.\ldots, f_{k}\right): R^{n} \times R^{m} \rightarrow R^{k}$ is said to be second order $(K, F)$ pseudoconvex at $u \in X$, for fixed $v$, if there exists sublinear function $F: X \times X \times R^{n} \rightarrow R, q \in R^{n}, x \in X, v \in Y$ such that

$$
\begin{aligned}
& -\left(F_{x, u}\left(\nabla_{u} f_{1}(u, v)+\nabla_{u u} f_{1}(u, v) q_{1}\right), \ldots,\right. \\
& \left.F_{x, u}\left(\nabla_{u} f_{k}(u, v)+\nabla_{u u} f_{k}(u, v) q_{k}\right)\right) \notin \operatorname{int} K \\
& \Longrightarrow-\left(f_{1}(x, v)-f_{1}(u, v)+\frac{1}{2} q_{1}^{T} \nabla_{u} f_{1}(u, v) q_{1}, \ldots,\right. \\
& \left.f_{k}(x, v)-f_{k}(u, v)+\frac{1}{2} q_{k}^{T} \nabla_{u} f_{k}(u, v) q_{k}\right) \notin \operatorname{int} K .
\end{aligned}
$$

Definition 12. A thrice differentiable function $f=$ $\left(f_{1}, f_{2}, \ldots, f_{k}\right): R^{n} \times R^{m} \rightarrow R^{k}$ is said to be second $\operatorname{order}(K$, $G)$-pseudoconvex at $y \in Y$, for fixed $v$, if there exists sublinear function $G: Y \times Y \times R^{m} \rightarrow R, p \in R^{m}, x \in X, v \in Y$ such that

$$
\begin{gathered}
-\left(G_{v, y}\left(\nabla_{y} f_{1}(x, y)+\nabla_{y y} f_{1}(x, y) p_{1}\right), \ldots,\right. \\
\left.G_{v, y}\left(\nabla_{y} f_{k}(x, y)+\nabla_{y y} f_{k}(x, y) p_{k}\right)\right) \notin \operatorname{int} K \\
\Longrightarrow-\left(f_{1}(x, v)-f_{1}(x, y)+\frac{1}{2} p_{1}^{T} \nabla_{y} f_{1}(x, y) p_{1}, \ldots,\right. \\
\left.f_{k}(x, v)-f_{k}(x, y)+\frac{1}{2} p_{k}^{T} \nabla_{y} f_{k}(x, y) p_{k}\right)
\end{gathered}
$$

$\notin \operatorname{int} K$.

Lemma 13 (generalized Schwartz inequality). Let $B$ be a positive semidefinite matrix of order $n$. Then, for all $x, w \in$ $R^{n}, x^{T} B w \leq\left(x^{T} B x\right)^{1 / 2}\left(w^{T} B w\right)^{1 / 2}$.

The equality holds if $B x=\lambda B w$ for some $\lambda \geq 0$.

\section{Wolfe Type Second Order Multiobjective Nondifferentiable Dual Programs}

We consider the following pair of second order Wolfe type nondifferentiable multiobjective programming problems with k-objective. 
Primal (SWP). Consider

$$
\begin{aligned}
& L(x, y, \lambda, w, p) \\
& =\text { Minimize }\left\{f_{i}(x, y)+\left(x^{T} B_{i} x\right)^{1 / 2}\right. \\
& -y^{T}\left[\nabla_{y} f_{i}(x, y)+\nabla_{y y} f_{i}(x, y) p\right] \\
& \left.-\frac{1}{2} p_{i}^{T}\left[\nabla_{y y} f_{i}(x, y) p_{i}\right], i=1,2, \ldots, k\right\} \\
& \text { Subject to } \quad-\sum_{i=1}^{k} \lambda_{i}\left[\nabla_{y} f_{i}(x, y)-D_{i} w_{i}+\nabla_{y y} f_{i}(x, y) p_{i}\right] \\
& \in C_{2}^{*} \\
& w_{i}^{T} D_{i} w_{i} \leq 1, \quad i=1,2, \ldots, k, \\
& x \in C_{1}, \quad w_{i} \in R^{m}, \\
& \lambda \in \operatorname{int} K^{*}, \quad \sum_{i=1}^{k} \lambda_{i}=1 \text {. }
\end{aligned}
$$

Dual (SWD). Consider

$$
\begin{aligned}
& M(u, v, \lambda, z, q) \\
& =\text { Maximize }\left\{f_{i}(u, u)-\left(v^{T} D_{i} v\right)^{1 / 2}\right. \\
& -u^{T}\left[\nabla_{u} f_{i}(u, v)+\nabla_{u u} f_{i}(u, v) q_{i}\right] \\
& \left.-\frac{1}{2} q_{i}^{T}\left[\nabla_{u u} f_{i}(u, v) q_{i}\right], i=1,2, \ldots, k\right\} \\
& \text { Subject to } \sum_{i=1}^{k} \lambda_{i}\left[\nabla_{u} f_{i}(u, v)+B_{i} z_{i}+\nabla_{u u} f_{i}(u, v) q_{i}\right] \\
& \in C_{1}^{*}, \\
& z_{i}^{T} B_{i} z_{i} \leq 1, \quad i=1,2, \ldots, k, \\
& v \in C_{2}, \quad z_{i} \in R^{n}, \\
& \lambda \in \operatorname{int} K^{*}, \quad \sum_{i=1}^{k} \lambda_{i}=1 \text {, }
\end{aligned}
$$

where

(1) $f=\left(f_{1}, f_{2}, \ldots, f_{k}\right): R^{n} \times R^{m} \rightarrow R^{k}$ is thrice differentiable function,

(2) $C_{1}$ and $C_{2}$ are closed convex cones in $R^{n}$ and $R^{m}$ with nonempty interiors, respectively,

(3) $C_{1}^{*}$ and $C_{2}^{*}$ are positive polar cones of $C_{1}$ and $C_{2}$, respectively,

(4) $K$ is a closed convex cone in $R^{k}$ with int $K \neq \phi$ and $R_{+}^{k} \subset K$,
(5) $q_{i}, z_{i}(i=1,2, \ldots, k)$ are vectors in $R^{n}$, and $p_{i}, w_{i}(i=$ $1,2, \ldots, k)$ are vectors in $R^{m}$

(6) $B_{i}$ and $D_{i}(i=1,2, \ldots, k)$ are positive semidefinite matrices of order $n$ and $m$, respectively.

Now we establish the following theorem.

Theorem 14 (weak duality theorem). Let $(x, y, \lambda, p)$ be a feasible solution for the primal (WP) and let $(u, v, \lambda, q)$ be a feasible solution for the dual (WD). Suppose there exist sublinear functional $F: X \times X \times R^{n} \rightarrow R$ and $G: Y \times Y \times R^{m} \rightarrow$ $R$ satisfying
(1) $F_{x, u}(a)-u^{T} a \geq 0$, for all $(x, u) \in C_{1} \times C_{1}, a \in C_{1}^{*}$,
(2) $G_{v, y}(b)-y^{T} b \geq 0$, for all $(v, y) \in C_{2} \times C_{2}, b \in C_{2}^{*}$.

Furthermore assume that, for each $i, f_{i}(\cdot, v)+(\cdot)^{T} B_{i} z_{i}$ is second order $(K, F)$-pseudoconvex at $u$ for fixed $v$ and $f_{i}(x, \cdot)+$ $(\cdot)^{T} D_{i} w_{i}$ is second order pseudoconcave at $y$ for fixed $x$ :

$$
\operatorname{Inf}(W P)-\operatorname{Sup}(W D) \in \operatorname{int} K \text {. }
$$

Proof. Since $(u, v, \lambda, q)$ is feasible solution for (WD), from dual constraint (5) we have

$$
a=\sum_{i=1}^{k} \lambda_{i}\left[\nabla_{u} f_{i}(u, v)+B_{i} z_{i}+\nabla_{u u} f_{i}(u, v) q_{i}\right] \in C_{1}^{*} .
$$

So

$u \in C_{1} \Longrightarrow u^{T} a$

$$
=u^{T}\left(\sum_{i=1}^{k} \lambda_{i}\left[\nabla_{u} f_{i}(u, v)+B_{i} z_{i}+\nabla_{u u} f_{i}(u, v) q_{i}\right]\right) \geq 0 .
$$

Again hypothesis (1) implies $F_{x, u}(a) \geq 0$. Consider

$$
\Longrightarrow F_{x, u}\left(\sum_{i=1}^{k} \lambda_{i}\left[\nabla_{u} f_{i}(u, v)+B_{i} z_{i}+\nabla_{u u} f_{i}(u, v) q_{i}\right]\right) \geq 0 \text {. }
$$

Since $F$ is sublinear with respect to third argument,

$$
\Longrightarrow \sum_{i=1}^{k} \lambda_{i} F_{x, u}\left(\left[\nabla_{u} f_{i}(u, v)+B_{i} z_{i}+\nabla_{u u} f_{i}(u, v) q_{i}\right]\right) \geq 0 .
$$

Since $\lambda \in \operatorname{int} K$, the above inequality can be written as

$$
\begin{aligned}
-\left(F_{x, u}\left(\left[\nabla_{u} f_{1}(u, v)+B_{1} z_{1}+\nabla_{u u} f_{1}(u, v) q_{1}\right]\right), \ldots,\right. \\
\left.F_{x, u}\left(\left[\nabla_{u} f_{k}(u, v)+B_{k} z_{k}+\nabla_{u u} f_{k}(u, v) q_{k}\right]\right)\right) \notin \operatorname{int} K .
\end{aligned}
$$

So second order $(K, F)$-pseudoconvexity of $f_{i}(\cdot, v)+(\cdot)^{T} B_{i} z_{i}$ at $u$ for fixed $v$ implies that

$$
-\left(\begin{array}{c}
f_{1}(x, v)+(x)^{T} B_{1} z_{1}-f_{1}(u, v)-(u)^{T} B_{1} z_{1} \\
+\frac{1}{2} q_{1}^{T} \nabla_{u u} f_{1}(u, v) q_{1}, \ldots, f_{k}(x, v)+(x)^{T} B_{k} z_{k} \\
-f_{k}(u, v)-(u)^{T} B_{k} z_{k}+\frac{1}{2} q_{k}^{T} \nabla_{u u} f_{k}(u, v) q_{k}
\end{array}\right) \notin \operatorname{int} K .
$$


This implies that, for $\lambda \in \operatorname{int} K$,

$$
\begin{gathered}
-\sum_{i=1}^{k} \lambda_{i}\left[f_{i}(x, v)+(x)^{T} B_{i} z_{i}-f_{i}(u, v)\right. \\
\left.-(u)^{T} B_{i} z_{i}-\frac{1}{2} q_{i}^{T} \nabla_{u u} f_{i}(u, v) q_{i}\right] \nsupseteq 0 \\
\Longrightarrow \sum_{i=1}^{k} \lambda_{i}\left[f_{i}(x, v)+(x)^{T} B_{i} z_{i}-f_{i}(u, v)\right. \\
\left.-(u)^{T} B_{i} z_{i}-\frac{1}{2} q_{i}^{T} \nabla_{u u} f_{i}(u, v) q_{i}\right] \geq 0 .
\end{gathered}
$$

Similarly $(u, v, \lambda, q)$ is feasible solution for (WD), so from primal constraint (1) we have

$$
b=-\sum_{i=1}^{k} \lambda_{i}\left[\nabla_{y} f_{i}(x, y)-D_{i} w_{i}+\nabla_{y y} f_{i}(x, y) p_{i}\right] \in C_{2}^{*} .
$$

So

$$
\begin{aligned}
y \in C_{2} & \Longrightarrow y^{T} b \\
=-y^{T}\left(\sum _ { i = 1 } ^ { k } \lambda _ { i } \left[\nabla_{y} f_{i}(x, y)\right.\right. & \\
& \left.\left.\quad-D_{i} w_{i}+\nabla_{y y} f_{i}(x, y) p_{i}\right]\right) \geq 0 .
\end{aligned}
$$

Again hypothesis (2) implies $G_{v, y}(b) \geq 0$. Consider

$$
\begin{aligned}
& \Longrightarrow G_{v, y}\left(-\sum_{i=1}^{k} \lambda_{i}\left[\nabla_{y} f_{i}(x, y)-D_{i} w_{i}+\nabla_{y y} f_{i}(x, y) p_{i}\right]\right) \\
& \quad \geq 0 .
\end{aligned}
$$

Since $G$ is sublinear with respect to third argument,

$$
\begin{aligned}
& \Longrightarrow \sum_{i=1}^{k} \lambda_{i} G_{v, y}\left(-\left[\nabla_{y} f_{i}(x, y)-D_{i} w_{i}+\nabla_{y y} f_{i}(x, y) p_{i}\right]\right) \\
& \quad \geq 0 .
\end{aligned}
$$

Since $\lambda \in \operatorname{int} K$, the above inequality can be written as

$$
\Longrightarrow-\left(\begin{array}{l}
G_{v, y}\left(-\left[\nabla_{y} f_{1}(x, y)-D_{1} w_{1}+\nabla_{y y} f_{1}(x, y) p_{1}\right]\right), \ldots \\
\ldots, G_{v, y}\left(-\left[\nabla_{y} f_{k}(x, y)-D_{k} w_{k}+\nabla_{y y} f_{k}(x, y) p_{k}\right]\right)
\end{array}\right) \notin \operatorname{int} K .
$$

So second order $(K, F)$-pseudoconcavity of $f_{i}(x, \cdot)-(\cdot)^{T} D_{i} w_{i}$ at $y$ for fixed $x$ implies that

$$
\begin{aligned}
& -\left(-\left[f_{1}(x, v)-(v)^{T} D_{1} w_{1}-f_{1}(x, y)+(y)^{T} D_{1} w_{1}\right.\right. \\
& \left.\quad+\frac{1}{2} p_{1}^{T} \nabla_{y y} f_{1}(x, y) p_{1}\right], \ldots, \\
& -\left[f_{k}(x, v)-(v)^{T} D_{k} z_{k}-f_{k}(x, y)+(y)^{T} D_{k} w_{k}\right. \\
& \left.\left.\quad+\frac{1}{2} p_{k}^{T} \nabla_{y y} f_{k}(x, y) p_{k}\right]\right) \\
& \notin \operatorname{int} K .
\end{aligned}
$$

This implies that, for $\lambda \in$ int $K$,

$$
\begin{gathered}
-\sum_{i=1}^{k} \lambda_{i}\left[-\left[f_{k}(x, v)-(v)^{T} D_{k} w_{k}-f_{k}(x, y)\right.\right. \\
\left.\left.+(y)^{T} D_{k} w_{k}+\frac{1}{2} p_{k}^{T} \nabla_{y y} f_{k}(x, y) p_{k}\right]\right] \nsupseteq 0 \\
\Longrightarrow \sum_{i=1}^{k} \lambda_{i}\left[-f_{i}(x, v)+(v)^{T} D_{i} w_{i}+f_{i}(x, y)\right. \\
\left.-(y)^{T} D_{i} w_{i}-\frac{1}{2} p_{i}^{T} \nabla_{y y} f_{i}(x, y) p_{i}\right] \geq 0 .
\end{gathered}
$$

Adding (30) and (37), we get

$$
\begin{gathered}
\Longrightarrow \sum_{i=1}^{k} \lambda_{i}\left[f_{i}(x, y)+(x)^{T} B_{i} z_{i}-(y)^{T} D_{i} w_{i}\right. \\
\left.-\frac{1}{2} p_{i}^{T} \nabla_{y y} f_{i}(x, y) p_{i}\right] \\
-\sum_{i=1}^{k} \lambda_{i}\left[f_{i}(u, v)-(v)^{T} D_{i} w_{i}+u^{T} B_{i} z_{i}\right. \\
\left.-\frac{1}{2} q_{i}^{T} \nabla_{u u} f_{i}(u, v) q_{i}\right] \geq 0 .
\end{gathered}
$$

Now from Schwartz inequality (Lemma 13), (17), and (21), we have

$$
\begin{aligned}
x^{T} B_{i} z_{i} \leq\left(x^{T} B_{i} x\right)^{1 / 2}\left(z_{i}^{T} B_{i} z_{i}\right)^{1 / 2} \leq & \left(x^{T} B_{i} x\right)^{1 / 2}, \\
i & =1,2, \ldots, k, \\
v^{T} D_{i} w_{i} \leq\left(v^{T} D_{i} v\right)^{1 / 2}\left(w_{i}^{T} D_{i} w_{i}\right)^{1 / 2} & \leq\left(v^{T} D_{i} v\right)^{1 / 2}, \\
& i=1,2, \ldots, k .
\end{aligned}
$$

Also from primal constraint (15), we have

$$
\sum_{i=1}^{k} \lambda_{i}\left[\nabla_{y} f_{i}(x, y)-D_{i} w_{i}+\nabla_{y y} f_{i}(x, y) p_{i}\right] \in C_{2}^{*}
$$


For $y \in C_{1}, y^{T}\left(\sum_{i=1}^{k} \lambda_{i}\left[\nabla_{y} f_{i}(x, y)-D_{i} w_{i}+\nabla_{y y} f_{i}(x, y) p_{i}\right]\right) \geq 0$

$$
\begin{aligned}
& -\sum_{i=1}^{k} \lambda_{i}\left[y^{T} D_{i} w_{i}\right] \\
& \quad \geq-y^{T}\left(\sum_{i=1}^{k} \lambda_{i}\left[\nabla_{y} f_{i}(x, y)+\nabla_{y y} f_{i}(x, y) p_{i}\right]\right) .
\end{aligned}
$$

Similarly from dual constraint (19), we have

$$
\begin{aligned}
\Longrightarrow & -\sum_{i=1}^{k} \lambda_{i}\left[u^{T} B_{i} z_{i}\right] \\
& \geq-u^{T}\left(\sum_{i=1}^{k} \lambda_{i}\left[\nabla_{u} f_{i}(u, v)+\nabla_{u u} f_{i}(u, v) q_{i}\right]\right) .
\end{aligned}
$$

Using (39), (41), and (42) in (38), we obtain that

$$
\begin{gathered}
\sum_{i=1}^{k} \lambda_{i}\left\{f_{i}(x, y)+\left(x^{T} B_{i} x\right)^{1 / 2}\right. \\
-y^{T}\left[\nabla_{y} f_{i}(x, y)+\nabla_{y y} f_{i}(x, y) p\right] \\
\left.-\frac{1}{2} p_{i}^{T}\left[\nabla_{y y} f_{i}(x, y) p_{i}\right]\right\} \\
-\sum_{i=1}^{k} \lambda_{i}\left\{f_{i}(u, u)-\left(v^{T} D_{i} v\right)^{1 / 2}\right. \\
-u^{T}\left[\nabla_{u} f_{i}(u, v)+\nabla_{u u} f_{i}(u, v) q_{i}\right] \\
\left.-\frac{1}{2} q_{i}^{T}\left[\nabla_{u u} f_{i}(u, v) q_{i}\right]\right\} \geq 0 \\
\Longrightarrow \operatorname{Inf}(\mathrm{WP})-\operatorname{Sup}(\mathrm{WD}) \in \operatorname{int} K .
\end{gathered}
$$

Theorem 15 (strong duality). Let $(\bar{x}, \bar{y}, \bar{\lambda}, \bar{w}, \bar{p})$ be weakly efficient solution of $(W P)$ such that

(i) $\nabla_{y y} f_{i}(\bar{x}, \bar{y})$ is nonsingular,

(ii) the matrix $\sum_{i=1}^{k} \lambda_{i}\left[\nabla_{y}\left(\nabla_{y y} f_{i}(\bar{x}, \bar{y}) \bar{p}\right)\right]$ is positive definite,

(iii) the set $\left\{\nabla_{y} f_{1}-C_{1} w_{1}, \ldots, \nabla_{y} f_{k}-C_{k} w_{k}\right\}$ is linearly independent,

(iv) $\sum_{i=1}^{k} \bar{\lambda}_{i}\left(\nabla_{y}\left(\nabla_{y y} f_{i} \bar{p}\right)\right) \bar{p} \notin \operatorname{span}\left\{\nabla_{y} f_{1}-C_{1} w_{1}, \ldots, \nabla_{y} f_{k}-\right.$ $\left.C_{k} w_{1}\right\} \backslash\{0\}$

Then there exist $\bar{z}_{i} \in R^{n}$ such that $(\bar{x}, \bar{y}, \bar{\lambda}, \bar{z}, \bar{q}=0)$ is feasible for (WD) and two objective values of (WP) and (WD) are equal. Also, if the hypotheses of Theorem 14 are satisfied for all feasible solution of (WP) and (WD), then $(\bar{x}, \bar{y}, \bar{\lambda}, \bar{z}, \bar{q}=0)$ is an efficient solution of (WD).

Proof. Since $(\bar{x}, \bar{y}, \bar{\lambda}, \bar{w}, \bar{p})$ is weakly efficient solution of (WD), by the Fritz-John necessary optimality condition on convex cone domain given in Bazaraa and Goode [20], there exist $\alpha \in K^{*}, \beta \in C_{2}, \gamma, \tau_{i} \in R_{+}$such that the following conditions are satisfied at $(\bar{x}, \bar{y}, \bar{\lambda}, \bar{w}, \bar{p})$ :

$$
\begin{aligned}
& (x-\bar{x})^{T} \\
& \times\left(\sum_{i=1}^{k} \alpha_{i}\left[\nabla_{x} f_{i}+B_{i} z_{i}\right]+\sum_{i=1}^{k} \lambda_{i}\left(\nabla_{y x} f_{i}\right)^{T}(\beta-\gamma \bar{y})\right. \\
& \left.\quad+\sum_{i=1}^{k} \lambda_{i}\left(\nabla_{x}\left(\nabla_{y y} f_{i} \bar{p}_{i}\right)\right)^{T}\left[\beta-\gamma \bar{y}-\alpha_{i} \bar{p}_{i}\right]\right)
\end{aligned}
$$

$\geq 0, \quad \forall x \in C_{1}$,

$$
\sum_{i=1}^{k}\left(\alpha_{i}-\gamma \lambda_{i}\right)\left(\nabla_{y} f_{i}-C_{i} w_{i}\right)
$$

$$
\begin{gathered}
+\sum_{i=1}^{k} \lambda_{i}\left(\nabla_{y y} f_{i}\right)^{T}[\beta-\gamma(\bar{y}+\bar{p})] \\
+\sum_{i=1}^{k} \lambda_{i}\left(\nabla_{y}\left(\nabla_{y y} f_{i} \bar{p}_{i}\right)\right)^{T}\left(\beta-\gamma \bar{y}-\frac{1}{2} \gamma \bar{p}_{i}\right)=0, \\
\sum_{i=1}^{k} \lambda_{i}\left(\nabla_{y y} f_{i}\right)^{T}\left(\beta-\gamma \bar{y}-\gamma \bar{p}_{i}\right)=0, \\
\sum_{i=1}^{k} \lambda_{i}\left(\nabla_{y} f_{i}-C_{i} w_{i}+\nabla_{y y} f_{i} \bar{p}_{i}\right)^{T} \beta=0, \\
x^{T} B_{i} z_{i}=\left(x^{T} B_{i} x\right)^{1 / 2}, \quad i=1,2, \ldots, k \\
\bar{\lambda}=\left(\bar{\lambda}_{1}, \bar{\lambda}_{2}, \ldots, \bar{\lambda}_{k}\right) \in \operatorname{int} K, \\
\alpha_{i} C_{i} \bar{y}+(\beta-\lambda \bar{y}) \lambda_{i} C_{i}=2 \tau_{i} C_{i} w_{i}, \\
\tau_{i}\left(\bar{w}_{i}^{T} C_{i} \bar{w}_{i}-1\right)=0 \\
\bar{z}_{i}^{T} B_{i} \bar{z}_{i} \leq 1 \\
(\alpha, \beta, \gamma, \tau) \geq 0 \\
(\alpha, \beta, \gamma, \tau) \neq 0 .
\end{gathered}
$$

Since $\nabla_{y y} f_{i}$ is nonsingular, (46) implies that $\beta=\gamma\left(\bar{y}+\bar{p}_{i}\right)$

$$
\beta=\gamma\left(\bar{y}+\bar{p}_{i}\right) .
$$

We claim that $\gamma>0$. Indeed if $\gamma=0$, then (55) implies $\beta=0$, which contradicts (54).

Hence

$$
\gamma>0 \text {. }
$$

Since $\gamma>0$, using (55) in (45), we get

$$
\sum_{i=1}^{k} \lambda_{i}\left[\nabla_{y}\left(\nabla_{y y} f_{i} \bar{p}\right)\right]^{T} \bar{p}_{i}=\frac{-2}{\gamma} \sum_{i=1}^{k}\left(\alpha_{i}-\gamma \lambda_{i}\right)\left[\nabla_{y} f_{i}-C_{i} w_{i}\right],
$$


which by hypothesis (ii) and (iv) yields

$$
\bar{p}_{i}=0, \quad i=1,2, \ldots, k .
$$

From (55) and (58), we obtain

$$
\beta=\gamma \bar{y}
$$

Using (58) and (59) and hypothesis (iii) in (45), we get

$$
\alpha=\gamma \lambda \text {. }
$$

Again using (58), (59), and (60) in (44), we get

$$
(x-\bar{x})^{T} \sum_{i=1}^{k} \lambda_{i}\left[\nabla_{x} f_{i}+B_{i} z_{i}\right] \geq 0, \quad \forall x \in C_{1} .
$$

Let $x \in C_{1}$. Then $x+\bar{x} \in C_{1}$ and so (61) implies

$$
\begin{aligned}
& (x)^{T} \sum_{i=1}^{k} \lambda_{i}\left[\nabla_{x} f_{i}+B_{i} z_{i}\right] \geq 0, \quad \forall x \in C_{1} \\
& \Longrightarrow \sum_{i=1}^{k} \lambda_{i}\left[\nabla_{x} f_{i}+B_{i} z_{i}\right] \in C_{1}^{*} .
\end{aligned}
$$

Also from (56), (59), and $\beta \in C_{2}$, we obtain

$$
\bar{y} \in C_{2} \text {. }
$$

Thus, from (52), (62), and (63), we obtain that $(\bar{x}, \bar{y}, \bar{z}, \bar{\lambda}, \bar{q}=$ $0)$ satisfies the dual constraints (19), (20), (21), and (22).

Thus $(\bar{x}, \bar{y}, \bar{z}, \bar{\lambda}, \bar{q}=0)$ is feasible for (WD).

Let $\left(2 \tau_{i} / \alpha_{i}\right)=t$; then $t \geq 0$. From (50) and (59), we get

$$
C_{i} \bar{y}=t C_{i} \bar{w}_{i}
$$

This is a condition of Schwartz inequality:

$$
\bar{y} C_{i} \bar{w}_{i}=\left(\bar{y}^{T} C_{i} \bar{w}_{i}\right)^{1 / 2}\left(\bar{w}_{i}^{T} C_{i} \bar{w}_{i}\right) .
$$

In case $\tau_{i}>0$, from (51) we get $\bar{w}_{i}^{T} C_{i} \bar{w}=1$. So (65) implies $\bar{y} C_{i} \bar{w}_{i}=\left(\bar{y}^{T} C_{i} \bar{w}_{i}\right)^{1 / 2}$.

In case $\tau_{i}=0$, we get $t=0$. So $C_{i} \bar{y}=0$. Hence $\bar{y} C_{i} \bar{w}_{i}=$ $\left(\bar{y}^{T} C_{i} \bar{w}_{i}\right)^{1 / 2}$. Thus in either case

$$
\bar{y} C_{i} \bar{w}_{i}=\left(\bar{y}^{T} C_{i} \bar{w}_{i}\right)^{1 / 2} .
$$

So using (48) and (66), we obtained that the two objective values are equal; that is,

$$
L(\bar{x}, \bar{y}, \bar{w}, \bar{\lambda}, \bar{p}=0)=M(\bar{x}, \bar{y}, \bar{z}, \bar{\lambda}, \bar{q}=0) .
$$

Now we claim that $(\bar{x}, \bar{y}, \bar{z}, \bar{\lambda}, \bar{q}=0)$ is an efficient solution of (WD). If this would not be the case, then there would exist a feasible solution $(\bar{u}, \bar{v}, \bar{z}, \bar{\lambda}, \bar{q}=0)$ such that

$$
\begin{aligned}
& M(\bar{x}, \bar{y}, \bar{z}, \bar{\lambda}, \bar{q}=0) \leq M(\bar{u}, \bar{v}, \bar{z}, \bar{\lambda}, \bar{q}=0) \\
& \Longrightarrow L(\bar{x}, \bar{y}, \bar{z}, \bar{\lambda}, \bar{q}=0) \leq M(\bar{u}, \bar{v}, \bar{z}, \bar{\lambda}, \bar{q}=0) .
\end{aligned}
$$

This is a contradiction to weak duality Theorem 14 .

Hence $(\bar{x}, \bar{y}, \bar{z}, \bar{\lambda}, \bar{q}=0)$ is efficient solution.
Theorem 16 (converse duality theorem). Let $(\bar{x}, \bar{y}, \bar{\lambda}, \bar{w}, \bar{p})$ be a weakly efficient solution of (WP) such that

(i) $\nabla_{u u} f_{i}(\bar{u}, \bar{v})$ is nonsingular,

(ii) the matrix $\sum_{i=1}^{k} \lambda_{i}\left[\nabla_{u}\left(\nabla_{u u} f_{i}(\bar{u}, \bar{v}) \bar{q}\right)\right]$ is positive definite,

(iii) the set $\left\{\nabla_{u} f_{1}+B_{1} z_{1}, \ldots, \nabla_{u} f_{k}+B_{k} z_{k}\right\}$ is linearly independent,

(iv) $\sum_{i=1}^{k} \bar{\lambda}_{i}\left(\nabla_{u}\left(\nabla_{u u} f_{i} \bar{q}\right)\right) \bar{q} \notin \operatorname{span}\left\{\nabla_{u} f_{1}+B_{1} z_{1}, \ldots, \nabla_{u} f_{k}+\right.$ $\left.B_{k} z_{k}\right\} \backslash\{0\}$.

Then there exist $\bar{w}_{i} \in R^{m}$ such that $(\bar{u}, \bar{v}, \bar{\lambda}, \bar{w}, \bar{p}=0)$ is feasible for (WD) and two objective values of (WP) and (WD) are equal. Also, if the hypotheses of Theorem 14 are satisfied for all feasible solution of (WP) and (WD), then $(\bar{u}, \bar{v}, \bar{\lambda}, \bar{w}, \bar{p}=0)$ is an efficient solution of (WD).

Proof. The proof follows on lines of Theorem 15.

\section{Wolfe Type Minimax Mixed Integer Programming}

Let $U$ and $V$ be two arbitrary sets of integers in $R^{n_{1}}(0 \leq$ $\left.n_{1} \leq n\right)$ and $R^{m_{1}}\left(0 \leq m_{1} \leq m\right)$, respectively. Throughout this section, we constrained some of the components of the vector variables $x \in R^{n}$ and $y \in R^{m}$ to belong to arbitrary sets of integers $U$ and $V$, respectively. Then we write $(x, y)=\left(x^{1}, x^{2}, y^{1}, y^{2}\right)$, where $x^{1}=\left(x_{1}, x_{2}, \ldots, x_{n}\right)$ and $y^{1}=$ $\left(y_{1}, y_{2}, \ldots, y_{n}\right) . x^{2}$ and $y^{2}$ are the vectors of the remaining components of $x$ and $y$, respectively.

Definition 17. Let $s^{1}, s^{2}, \ldots, s^{r}$ be elements of an arbitrary vector space. A vector function $\theta\left(s^{1}, s^{2}, \ldots, s^{r}\right)$ will be called additively separable with respect to $s^{1}$, if there exist vector function $\theta_{1}\left(s^{1}\right)$ (independent of $\left.s^{2}, \ldots, s^{r}\right)$ and $\theta_{2}\left(s^{2}, \ldots, s^{r}\right.$ ) (independent of $\left.s^{1}\right)$ such that $\theta\left(s^{1}, s^{2}, \ldots, s^{r}\right)=\theta_{1}\left(s^{1}\right)+$ $\theta_{2}\left(s^{2}, \ldots, s^{r}\right)$.

We consider the following pair of Wolfe type nondifferentiable minimax mixed integer symmetric primal and dual programs:

Primal (WIP). Consider

$$
\begin{aligned}
\max _{x^{1}} \min _{x^{2}, y, w, p}\{ & f_{i}(x, y)+\left(\left(x^{2}\right)^{T} B_{i} x\right)^{1 / 2} \\
& -\left(y^{2}\right)^{T}\left[\nabla_{y^{2}} f_{i}(x, y)+\nabla_{y^{2} y^{2}} f_{i}(x, y) p\right] \\
& \left.-\frac{1}{2} p_{i}^{T}\left[\nabla_{y^{2} y^{2}} f_{i}(x, y) p_{i}\right], i=1,2, \ldots, k\right\}
\end{aligned}
$$

Subject to $-\sum_{i=1}^{k} \lambda_{i}\left[\nabla_{y^{2}} f_{i}(x, y)-D_{i} w_{i}+\nabla_{y^{2} y^{2}} f_{i}(x, y) p_{i}\right]$ $\in C_{2}^{*}$, 


$$
\begin{gathered}
w_{i}^{T} D_{i} w_{i} \leq 1, \quad i=1,2, \ldots, k \\
x^{2} \in C_{1}, \quad x^{1} \in U, \quad y^{1} \in V, \quad w_{i} \in R^{m}, \\
\lambda \in \operatorname{int} K^{*}, \quad \sum_{i=1}^{k} \lambda_{i}=1 .
\end{gathered}
$$

Dual (WID). Consider

$$
\begin{aligned}
& \min _{v^{1}} \max _{u, v^{2}, z}\left\{f_{i}(u, v)-\left(\left(v^{2}\right)^{T} D_{i} v\right)^{1 / 2}\right. \\
& +\left(u^{2}\right)^{T}\left[\nabla_{u^{2}} f_{i}(u, v)+\nabla_{u^{2} u^{2}} f_{i}(u, v) q_{i}\right] \\
& \left.-\frac{1}{2} q_{i}^{T}\left[\nabla_{u^{2} u^{2}} f_{i}(u, v) q_{i}\right], i=1,2, \ldots, k\right\} \\
& \in C_{1}^{*}, \\
& z_{i}^{T} B_{i} z_{i} \leq 1, \quad i=1,2, \ldots, k, \\
& v^{2} \in C_{2}, \quad u^{1} \in U, \quad v^{1} \in V, \quad z_{i} \in R^{n}, \\
& \lambda \in \operatorname{int} K^{*}, \quad \sum_{i=1}^{k} \lambda_{i}=1,
\end{aligned}
$$

where

(1) $f=\left(f_{1}, f_{2}, \ldots, f_{k}\right): R^{n} \times R^{m} \rightarrow R^{k}$ is thrice differentiable function,

(2) $C_{1}$ and $C_{2}$ are closed convex cones in $R^{n-n_{1}}$ and $R^{m-m_{1}}$ with nonempty interiors, respectively,

(3) $C_{1}^{*}$ and $C_{2}^{*}$ are positive polar cones of $C_{1}$ and $C_{2}$, respectively,

(4) $K$ is a closed convex cone in $R^{k}$ with int $K \neq \phi$ and $R_{+}^{k} \subset K$,

(5) $q_{i}, z_{i}(i=1,2, \ldots, k)$ are vectors in $R^{n}$, and $p_{i}, w_{i}(i=$ $1,2, \ldots, k)$ are vectors in $R^{m}$

(6) $B_{i}$ and $D_{i}(i=1,2, \ldots, k)$ are positive semidefinite matrices of order $n-n_{1}$ and $m-m_{1}$, respectively.

Theorem 18 (symmetric duality). Let $(\bar{x}, \bar{y}, \bar{\lambda}, \bar{w}, \bar{p})$ be a weakly efficient solution of (WIP). Also

(i) $f(x, y)$ is additively separable with respect to $x^{1}$ or $y^{1}$; that is, $f_{i}\left(x^{1}, x^{2}, y\right)=f_{i 1}\left(x^{1}\right)+f_{i 2}\left(x^{2}, y\right)$,

(ii) $f(x, y)$ is thrice differentiable in $x^{2}$ and $y^{2}$,

(iii) $\nabla_{y^{2} y^{2}} f(\bar{x}, \bar{y})$ is nonsingular,

(iv) the vector $\bar{p}^{T} \nabla_{y^{2}}\left(\nabla_{y^{2} y^{2}} f(\bar{x}, \bar{y}) \bar{p}\right)=0 \Rightarrow \bar{p}=0$. Furthermore, for any feasible solution $(x, y, \lambda, w, p)$ in (WIP) and for any feasible solution $(u, v, \lambda, z, q)$ in (WID), suppose there exist functional $F^{\prime}: X^{\prime} \times X^{\prime} \times$ $R^{n-n_{1}} \rightarrow R$ and $G^{\prime}: Y^{\prime} \times Y^{\prime} \times R^{m-m_{1}} \rightarrow R$ such that

(v) $\left(f_{12}\left(u^{2}, v\right)+\left(u^{2}\right)^{T} B_{1} z_{1}, \ldots, f_{k 2}\left(u^{2}, v\right)+\left(u^{2}\right)^{T} B_{k} z_{k}\right)$ is second order $\left(K, F^{\prime}\right)$ pseudoconvex at $u^{2}$ with respect to $q \in R^{n-n_{1}}$ for each $\left(u^{1}, v\right)$ and $\left(f_{12}\left(x, y^{2}\right)-\right.$ $\left.\left(y^{2}\right)^{T} D_{1} w_{1}, \ldots, f_{12}\left(x, y^{2}\right)-\left(y^{2}\right)^{T} D_{1} w_{1}\right)$ is second $\operatorname{order}\left(K, G^{\prime}\right)$ pseudoconcave at $y^{2}$ with respect to $p \in$ $R^{m-m_{1}}$ for each $\left(x, y^{1}\right)$,

(vi) $F_{x^{2}, u^{2}}^{\prime}(a)-\left(u^{2}\right)^{T} a \geq 0$, for all $a \in C_{1}^{*}$, and $G_{v^{2}, y^{2}}^{\prime}(b)-$ $\left(y^{2}\right)^{T} b \geq 0$, for all $b \in C_{2}^{*}$.

Then there exist $z \in R^{n-n_{1}}$ such that $(\bar{x}, \bar{y}, \bar{\lambda}, \bar{z}, \bar{q}=0)$ is efficient solution for dual and optimal values (WIP) and (WID) are equal.

Proof. Let

$$
\begin{aligned}
s=\max _{x^{1}} \min _{x^{2}, y, w}\{ & f_{i}(x, y)+\left(\left(x^{2}\right)^{T} B_{i} x^{2}\right)^{1 / 2}-\left(y^{2}\right)^{T} \\
& \times\left[\nabla_{y^{2}} f_{i}(x, y)+\nabla_{y^{2} y^{2}} f_{i}(x, y) p_{i}\right] \\
& -\frac{1}{2} p_{i}^{T} \nabla_{y^{2} y^{2}} f_{i}(x, y) p_{i},
\end{aligned}
$$

$$
\begin{gathered}
i=1,2, \ldots, k ;(x, y, w, p) \in S\}, \\
t=\min _{v^{1}} \max _{u, v^{2}, z}\left\{f_{i}(u, v)-\left(\left(v^{2}\right)^{T} C_{i} v^{2}\right)^{1 / 2}\right. \\
+\left(u^{2}\right)^{T}\left[\nabla_{u^{2}} f_{i}(u, v)+\nabla_{u^{2} u^{2}} f_{i}(u, v) q_{i}\right] \\
-\frac{1}{2} q_{i}^{T} \nabla_{u^{2} u^{2}} f_{i}(u, v) q_{i}, \\
i=1,2, \ldots, k ;(u, v, z, q) \in T\},
\end{gathered}
$$

where $S$ and $T$ are a feasible region of primal (WIP) and dual (WID), respectively.

Since $f_{i}(x, y)$ is additively separable with respect to $x^{1}$ or $y^{1}$ (say with respect to $x^{1}$ ) from definition, it follows that $f_{i}(x, y)=f_{i 1}\left(x^{1}\right)+f_{i 2}\left(x^{2}, y\right)$

Therefore

$$
\begin{aligned}
\nabla_{y^{2}} f_{i}(x, y) & =\nabla_{y^{2}} f_{i 2}\left(x^{2}, y\right), \nabla_{y^{2} y^{2}} f_{i}(x, y) \\
& =\nabla_{y^{2} y^{2}} f_{i 2}\left(x^{2}, y\right) .
\end{aligned}
$$


So the primal (WIP) can be written as

$$
\begin{aligned}
s=\max _{x^{1}} \min _{x^{2}, y, w}\left\{f_{i 1}\right. & \left(x^{1}\right)+f_{i 2}\left(x^{2}, y\right) \\
+ & \left(\left(x^{2}\right)^{T} B_{i} x^{2}\right)^{1 / 2}-\left(y^{2}\right)^{T} \\
& \times\left[\nabla_{y^{2}} f_{i 2}(x, y)+\nabla_{y^{2} y^{2}} f_{i 2}(x, y) p_{i}\right] \\
& -\frac{1}{2} p_{i}^{T} \nabla_{y^{2} y^{2}} f_{i 2}\left(x^{2}, y\right) p_{i}, \\
& i=1,2, \ldots, k ;(x, y, w, p) \in S\}
\end{aligned}
$$

or

$$
s=\max _{x^{1}} \min _{y^{1}}\left\{f_{i 1}\left(x^{1}\right)+\varphi_{i}\left(y^{1}\right) ; x^{1} \in U, y^{1} \in V\right\}
$$

where $\left(\mathrm{WIP}_{0}\right)$ :

$$
\begin{aligned}
& \varphi_{i}\left(y^{1}\right) \\
& =\min _{x^{2}, y^{2}, w}\left\{f_{i 2}\left(x^{2}, y\right)+\left(\left(x^{2}\right)^{T} B_{i} x^{2}\right)^{1 / 2}-\left(y^{2}\right)^{T}\right. \\
& \times\left[\nabla_{y^{2}} f_{i 2}(x, y)+\nabla_{y^{2} y^{2}} f_{i 2}(x, y) p_{i}\right] \\
& \left.-\frac{1}{2} p_{i}^{T} \nabla_{y^{2} y^{2}} f_{i 2}\left(x^{2}, y\right) p_{i}\right\} \\
& \text { Subject to } \quad-\sum_{i=1}^{k} \lambda_{i}\left[\nabla_{y^{2}} f_{i 2}\left(x^{2}, y\right)-C_{i} w_{i}\right. \\
& \left.+\nabla_{y^{2} y^{2}} f_{i 2}\left(x^{2}, y\right) p_{i}\right] \in C_{2}^{*}, \\
& \left(w_{i}\right)^{T} C_{i} w_{i} \leq 1, \quad i=1,2, \ldots, k \\
& \lambda \in \operatorname{int} K^{*}, \quad \sum_{i=1}^{k} \lambda_{i}=1, \\
& x^{2} \in C_{1}, \quad w_{i} \in R^{m-m_{1}}, \quad p_{i} \in R^{m-m_{1}} .
\end{aligned}
$$

Similarly the dual (WID) can be written as

$$
t=\min _{v^{1}} \max _{u^{1}}\left\{f_{i 1}\left(u^{1}\right)+\theta\left(v^{1}\right), u^{1} \in U, v^{1} \in V\right\}
$$

where $\left(\mathrm{WID}_{0}\right)$ :

$$
\begin{aligned}
\theta_{i}\left(v^{1}\right)=\max _{u^{2}, v^{2}, z, q}\{ & f_{i 2}\left(u^{2}, v\right) \\
& -\left(\left(v^{2}\right)^{T} C_{i} v^{2}\right)^{1 / 2}-\left(u^{2}\right)^{T} \\
& \times\left[\nabla_{u^{2}} f_{i 2}(u, v)+\nabla_{u^{2} u^{2}} f_{i 2}(u, v) q_{i}\right] \\
& \left.-\frac{1}{2} q_{i}^{T} \nabla_{u^{2} u^{2}} f_{i 2}\left(u^{2}, v\right) q_{i}\right\}
\end{aligned}
$$

For any given $y^{1}$ and $v^{1}$, programs $\left(\mathrm{WIP}_{0}\right)$ and $\left(\mathrm{WID}_{0}\right)$ are a pair of Wolfe type second order nondifferentiable multiobjective symmetric dual programs studied in Section 3 and hence in view of hypothesis (ii)-(vi), Theorems 14 and 15 become applicable. Therefore $y^{1}=\bar{y}^{1}=v^{1}$, and we obtain $\bar{q}=0, \varphi\left(y^{1}\right)=\theta\left(\bar{y}^{1}\right)$. So the two optimal values are equal and $(\bar{x}, \bar{y}, \bar{\lambda}, \bar{z}, \bar{q}=0)$ is an efficient solution for the dual.

\section{Special Cases}

(i) If $B_{i}=D_{i}=0, k=1$, then the problems (SWP) and (SWD) can be reduced to the problem proposed by Gulati et al. [14] as follows.

Primal (WP).

$$
\begin{array}{r}
\text { Minimize } f(x, y)-y^{T}\left[\nabla_{y} f(x, y)+\nabla_{y y} f(x, y) p\right] \\
-\frac{1}{2} p^{T}\left[\nabla_{y y} f(x, y) p\right] \\
\text { Subject to }-\left[\nabla_{y} f_{i}(x, y)+\nabla_{y y} f_{i}(x, y) p_{i}\right] \in C_{2}^{*}, \\
x \in C_{1} .
\end{array}
$$

Dual (WD).

Maximize $f(u, u)+u^{T}\left[\nabla_{u} f(u, v)+\nabla_{u u} f(u, v) q\right]$

$$
-\frac{1}{2} q^{T}\left[\nabla_{u u} f(u, v) q\right]
$$

Subject to $\nabla_{u} f(u, v)+\nabla_{u u} f(u, v) q \in C_{1}^{*}$,

$$
v \in C_{2} \text {. }
$$


(ii) If $k=1, C_{1}=R_{+}^{n}, C_{2}=R_{+}^{m},\left(x^{T} B x\right)^{1 / 2}=s\left(x \mid C^{\prime}\right)$, and $\left(y^{T} D y\right)^{1 / 2}=s\left(y \mid D^{\prime}\right)$, where $C^{\prime}=\left\{B x \mid x^{T} B x \leq 1\right\}$, $D^{\prime}=\left\{D y \mid y^{T} D y \leq 1\right\}$, then the problems (SWP) and (SWD) can be reduced to the problem proposed by Yang et al. [12].

Primal (WP).

$$
\begin{aligned}
\text { Minimize } & f(x, y)+s\left(x \mid C^{\prime}\right) \\
& -y^{T}\left[\nabla_{y} f(x, y)+\nabla_{y y} f(x, y) p\right] \\
& -\frac{1}{2} p^{T}\left[\nabla_{y y} f(x, y) p\right]
\end{aligned}
$$

Subject to $\nabla_{y} f(x, y)-z+\nabla_{y y} f(x, y) p \leq 0$,

$$
x \geq 0, \quad z \in D^{\prime} .
$$

$\operatorname{Dual}(W D)$.

$$
\begin{array}{ll}
\text { Maximize } & f(u, u)-s\left(v \mid D^{\prime}\right) \\
& -u^{T}\left[\nabla_{u} f(u, v)+\nabla_{u u} f(u, v) q\right] \\
& -\frac{1}{2} q^{T}\left[\nabla_{u u} f(u, v) q\right] \\
\text { Subject to } & \nabla_{u} f(u, v)+\nabla_{u u} f(u, v) q \geq 0, \\
& v \geq 0, \quad w \in C^{\prime} .
\end{array}
$$

\section{Conflict of Interests}

The author declares that there is no conflict of interests regarding the publication of this paper.

\section{References}

[1] V. Pareto, Course D'economic Politique, Lausanne, Raye, Saint Lucia, 1986.

[2] H. W. Kuhn and A. W. Tucker, "Nonlinear programming," in Proceedings of the 2nd Berkeley Symposium on Mathematical Statistics and Probability, pp. 481-492, University of California Press, Berkeley, Calif, USA, 1951.

[3] A. M. Geoffrion, "Proper efficiency and the theory of vector maximization," Journal of Mathematical Analysis and Applications, vol. 22, pp. 618-630, 1968.

[4] W. S. Dorn, "A symmetric dual theorem for quadratic programs," Journal of the Operations Research Society of Japan, vol. 2, pp. 93-97, 1960.

[5] G. B. Dantzig, E. Eisenberg, and R. W. Cottle, "Symmetric dual nonlinear programs," Pacific Journal of Mathematics, vol. 15, pp. 809-812, 1965.

[6] B. Mond, "Second order duality for nonlinear programs," Opsearch, vol. 11, no. 2-3, pp. 90-99, 1974.

[7] B. Mond and T. Weir, "Generalized concavity and duality," in Generalized Concavity in Optimization and Economics, S. Schaible and W. T. Ziemba, Eds., pp. 263-279, Academic Press, New York, NY, USA, 1981.

[8] O. L. Mangasarian, "Second- and higher-order duality in nonlinear programming," Journal of Mathematical Analysis and Applications, vol. 51, no. 3, pp. 607-620, 1975.
[9] C. R. Bector and S. Chandra, "Generalized bonvex function and second order duality in mathematical programming," Research Report 85-2, The University of Manitoba, Winnipeg, Canada, 1985.

[10] G. Devi, "Symmetric duality for nonlinear programming problem involving $\eta$-bonvex functions," European Journal of Operational Research, vol. 104, no. 3, pp. 615-621, 1998.

[11] S. Khurana, "Symmetric duality in multiobjective programming involving generalized cone-index functions," European Journal of Operational Research, vol. 165, no. 3, pp. 592-597, 2005.

[12] X. M. Yang, X. Q. Yang, K. L. Teo, and S. H. Hou, "Second order symmetric duality in non-differentiable multiobjective programming with F-convexity," European Journal of Operational Research, vol. 164, no. 2, pp. 406-416, 2005.

[13] X. M. Yang, X. Q. Yang, K. L. Teo, and S. H. Hou, "Multiobjective second-order symmetric duality with $F$-convexity," European Journal of Operational Research, vol. 165, no. 3, pp. 585-591, 2005.

[14] T. R. Gulati, S. K. Gupta, and I. Ahmad, "Second-order symmetric duality with cone constraints," Journal of Computational and Applied Mathematics, vol. 220, no. 1-2, pp. 347-354, 2008.

[15] T. R. Gulati and Geeta, "Mond-Weir type second-order symmetric duality in multiobjective programming over cones," Applied Mathematics Letters, vol. 23, no. 4, pp. 466-471, 2010.

[16] T. R. Gulati and K. Verma, "Nondifferentiable multiobjective Wolfe type symmetric duality under invexity," in Proceedings of the International Conference on Soft Computing for Problem Solving, vol. 1, pp. 347-354, 2012.

[17] S. K. Gupta and N. Kailey, "Nondifferentiable multiobjective second-order symmetric duality," Optimization Letters, vol. 5, no. 1, pp. 125-139, 2011.

[18] S. K. Gupta and N. Kailey, "Second-order multiobjective symmetric duality involving cone-bonvex functions," Journal of Global Optimization, vol. 55, no. 1, pp. 125-140, 2013.

[19] H. Saini and T. R. Gulati, "Nondifferentiable multiobjective symmetric duality with F-convexity over cones," Nonlinear Analysis: Theory, Methods \& Applications, vol. 74, no. 5, pp. 15771584, 2011.

[20] M. S. Bazaraa and J. J. Goode, "On symmetric duality in nonlinear programming," Operations Research, vol. 21, pp. 1-9, 1973. 


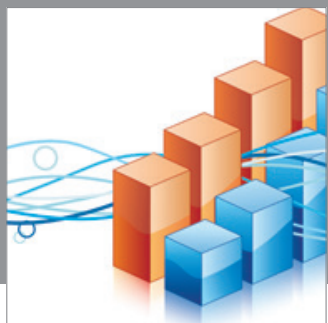

Advances in

Operations Research

mansans

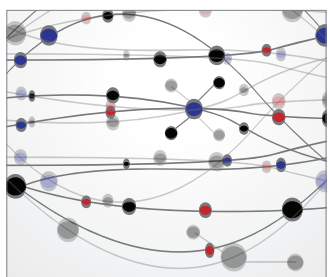

The Scientific World Journal
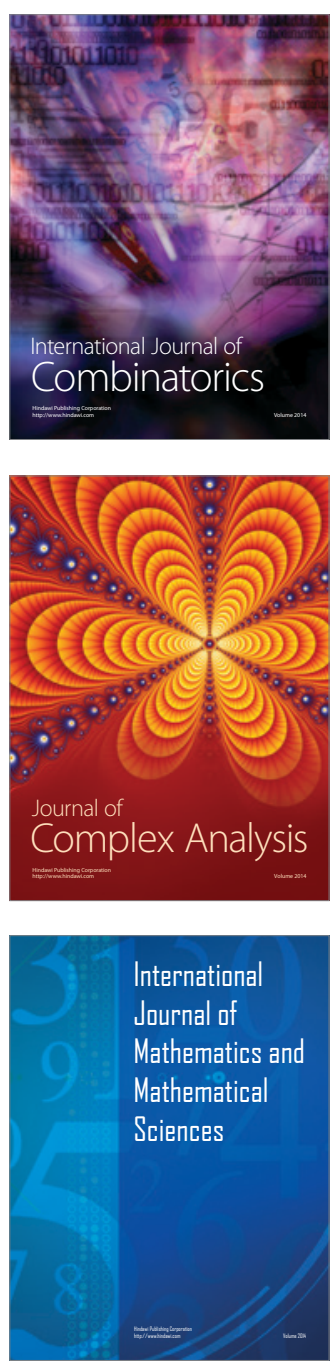
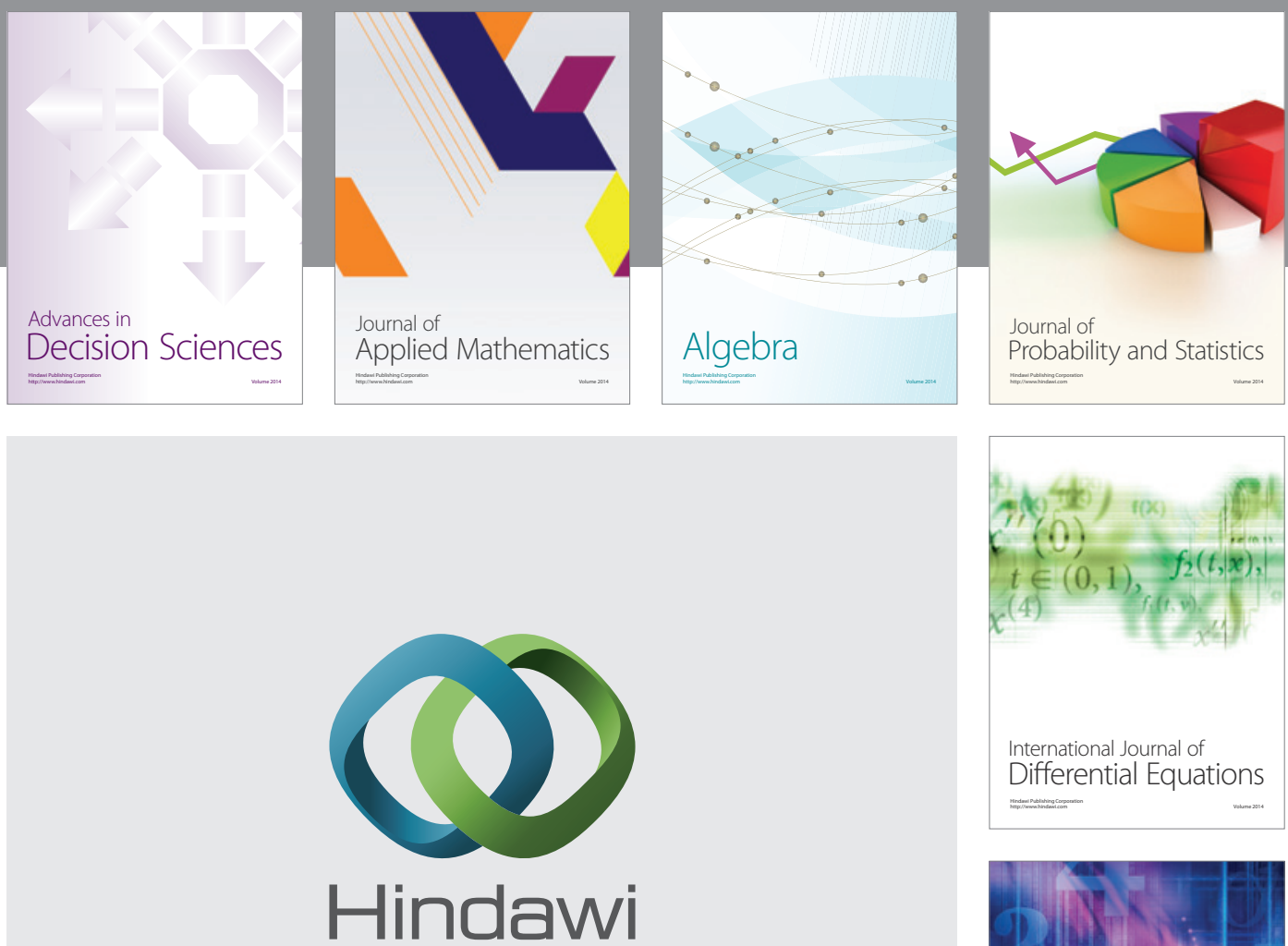

Submit your manuscripts at http://www.hindawi.com
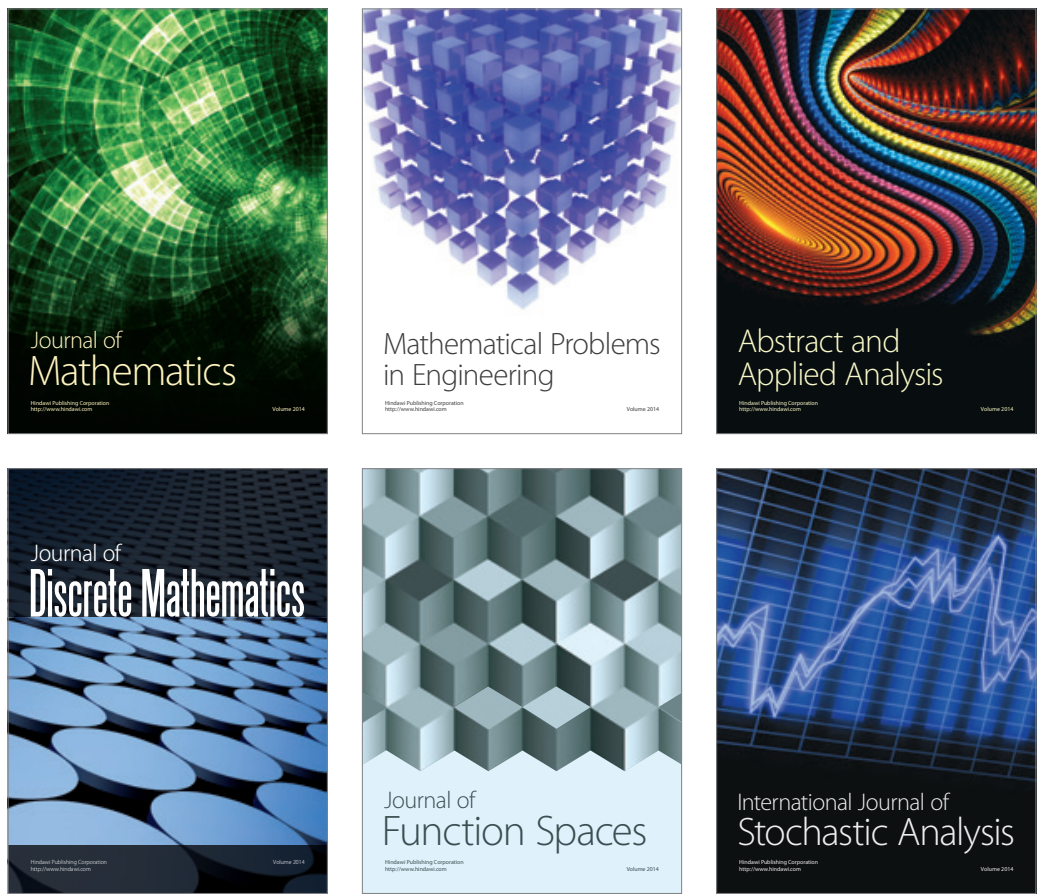

Journal of

Function Spaces

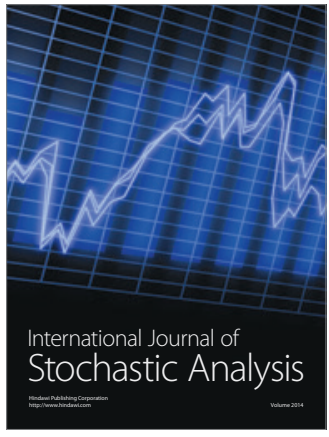

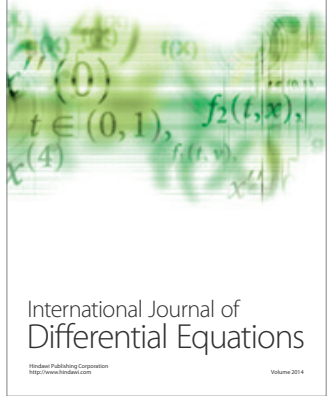
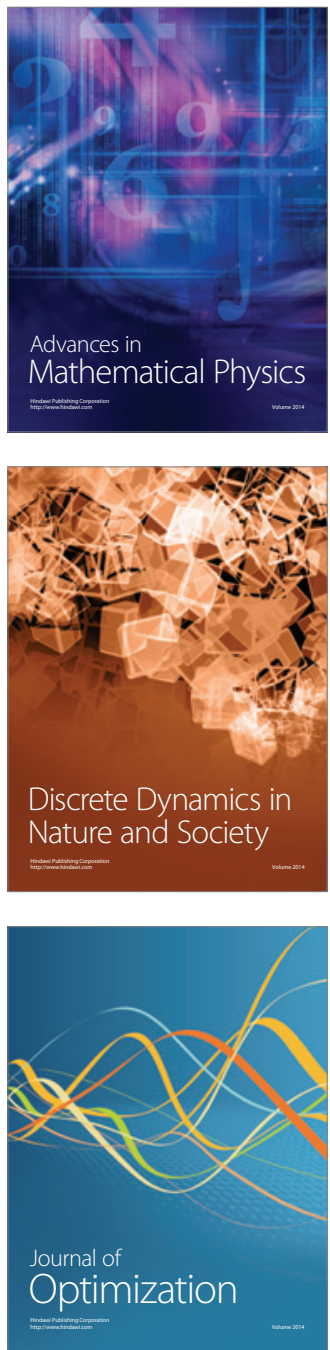